$\operatorname{IMSC} / 97 / 03 / 10$

\title{
Fundamental Strings and D-strings in the IIB Matrix Model
}

\author{
B. Sathiapalan \\ Institute of Mathematical Sciences \\ Taramani \\ Chennai 600113 \\ INDIA
}

April 26, 2018

\begin{abstract}
The matrix model for IIB Superstring proposed by Ishibashi, Kawai, Kitazawa and Tsuchiya is investigated. Consideration of planar and non-planar diagrams suggests that the large $\mathrm{N}$ perturbative expansion is consistent with the double scaling limit proposed by the above authors. We write down a Wilson loop that can be interpreted as a fundamental string vertex operator. The one point tadpole in the presence of a D-string has the right form and this can be viewed as a matrix model derivation of the boundary conditions that define a D-string. We also argue that if world sheet coordinates $\sigma$ and $\tau$ are introduced for the fundamental string, then the conjugate variable $\frac{d}{d \sigma}$ and $\frac{d}{d \tau}$ can be interpreted as the D-string world sheet coordinates. In this way the $S L(2 Z)$ duality group of the IIB superstring becomes identified with the symplectic group acting on $(p, q)$.
\end{abstract}




\section{Introduction}

A large N supersymmetric Yang-Mills (SSYM) matrix model in zero space and one time has been proposed as a non-perturbative definition of M-theory in the light cone gauge [1]. Motivated by this proposal, Ishibashi, Kawai, Kitazawa and Tsuchiya (IKKT) have proposed a reduced (zero space and no time) large N SSYM as a non perturbative definition of type IIB string theory [2]. While it is not yet clear whether there is any computational advantage, conceptually the matrix model approach seems very rich and fertile. In particular a host of non perturbative phenomena involving pbranes and D-p-branes can be incorporated into the formalism without much difficulty. Fundamental strings do not have a preferred status in these models (indeed they are hard to find), which is a viewpoint that has recently become popular. Thus these propoals merit serious study. Moreover, while the idea that large N Yang Mills theory is a string theory, is an old one, the proposals in [1, 2] are probably the most precise and concrete that have so far been made. Recently several papers have appeared investigating the IIA model [四, 3, 5, 6] and the IIB model [0, 8, 11, 9, 10].

In this paper we focus our attention on the type IIB theory of [2] and present some results that provide further evidence for their proposal. Taking their proposal that the Wislon loop represents the fundamental string, at face value (this is consistent with the old ideas of Yang Mills theory being a string theory), we study the genus expansion of the reduced model using the ideas of quenched Eguchi-Kawai models. We find that a double scaling limit very similar to that proposed by IKKT, does, in fact, define a sensible expansion. We then introduce string world sheet coordinates $\sigma$ and $\tau$ to recover the standard string theory world sheet action (in an approximation that neglects the quartic terms) in light cone gauge, and we are also able to show that appropriately constructed Wilson loops behave like vertex operators. In addition, we find that there is a symmetry between $(\sigma, \tau)$ and the conjugate variables $\left(\partial_{\sigma}, \partial_{\tau}\right)$. Since one set is as good as the other, an obvious interpretation is that the conjugate variables represent the coordinates of the D-string. This is also consistent with the idea that a solution of the equations of motion represent D-strings and that fluctuations of the D-string solution represent fundamental strings. This is analogous to the fact that in field theories, classical solutions represent solitons and the field quanta are then small fluctuations about the classical solution. But now, 
because of the complete symmetry between $(\sigma, \tau)$ and $\left(\partial_{\sigma}, \partial_{\tau}\right)$ the string and the D-string are on an equal footing and the $S L(2, Z)$ symmetry of type IIB is then nothing more than the symplectic group acting on $(p, q)$ i.e.

$$
\left(\begin{array}{l}
p^{\prime} \\
q^{\prime}
\end{array}\right)=\left(\begin{array}{ll}
a & b \\
c & d
\end{array}\right)\left(\begin{array}{l}
p \\
q
\end{array}\right)
$$

We then look at the one point function of the Wilson loop in the presence of the D-string and check that it reproduces the result of [2]. In the usual formalism, this result is a direct consequence of the boundary conditions on the open string that define a D-string [20]. Thus we can view this calculation as a matrix model derivation of the boundary conditions.

This paper is organized as follows. In section II we discuss the diagrammatic expansion of the reduced model and check the double scaling proposal. In section III we discuss in some detail the introduction of the world sheet coordinates for the fundamental (' $\mathrm{F}$ ')string and the D-string and argue that the symplectic group should be identified with the $\mathrm{SL}(2, \mathrm{Z})$ duality of IIB strings. We also study the spectrum and identify the $(1, \mathrm{~m})$ strings. In section IV we study some properties of the Wilson loop, namely the two point function in flat space and the one point function in the presence of a classical D-string background. We conclude with some comments in Section V. 


\section{Perturbation Theory with the Reduced Model}

Reduced models [12, 13, 14, 15, 16] were originally proposed as a large $\mathrm{N}$ approximation to the full theory, the main point being that the planar diagrams give the same result to leading order in $\frac{1}{N}$ as in the full theory. In the present context the reduced model at infinite $N$ T is being taken as the defining theory. Therefore, unlike in the early days, it makes sense to consider the subleading non-planar diagrams of the theory and check whether there is some kind of double scaling limit that can make sense. In the original context the perturbation series in Yang-Mills is of the form $\left(g^{2} N\right)^{\frac{V_{3}}{2}+V_{4}}\left(N^{2}\right)^{1-g}$. Here $V_{3}$ and $V_{4}$ are the numbers of cubic and quartic vertices respectively, and $g$ is the genus. The genus expansion is labelled by powers of $\frac{1}{N}$. Clearly in the reduced model, since we need to take $N \rightarrow \infty$ and not just large, this would not work. Thus there must be some other double scaling at work here. The idea that the reduced model perturbation could define a string in some double scaling limit has been proposed earlier [17].

In 22 a double scaling limit has been proposed, based on comparison with the classical action of the D-string. Below we consider the perturbation expansion of this model. We find that the double scaling limit proposed in [2] is the correct one. We follow the prescription of [15, 16]. Since we are only doing some simple counting and are not going to actually evaluate any diagrams, the details of the vertices are not important. The action of the SSYM is

$$
S=\int d^{10} x \frac{1}{2 g^{2}}\left[F_{\mu \nu}\right]^{2}+\text { Fermionic }
$$

Note that if we assign the usual canonical dimension of one to $A$ (in mass units), then $g$ has dimensions of $[\text { mass }]^{-3}$ or $[\text { length }]^{3}$. Thus we can write

$$
g^{2}=\frac{g_{0}^{2}}{N}=\frac{\lambda^{2}}{N}[m]^{-6}
$$

We have factored out $\frac{1}{N}$, in anticipation of the fact that $\left(g^{2} N\right)$ is usually held fixed. Here " $m$ " is some finite mass scale the theory is assumed to possess. In the reduced model we write

$$
S_{\text {Reduced }}=\left(\frac{2 \pi}{\Lambda}\right)^{d} \frac{1}{2 g^{2}}\left[A_{\mu}, A_{\nu}\right]^{2}+\text { Fermionic }
$$

\footnotetext{
${ }^{1} N$ needs to be literally infinite if one is to get anything non trivial that looks like the D-string of the continuum theory in some non zero space time dimension.
} 
following [15, 16]. $\left[\frac{2 \pi}{\Lambda}\right]^{d}$ can be taken as the volume of a basic cell, where $\Lambda$ is a momentum cutoff. Actually in this model, since the eigenvalues of $A$ represent position, $\Lambda$ should be interpreted as an infrared cutoff in the real space-time sense. Neverthelss, the mathematical manipulations are the same.

In order to do perturbation theory we assume that

$$
A_{\mu}=P_{\mu}+a_{\mu}
$$

" $P_{\mu}$ " can be thought of as the vev of $A_{\mu}$ and this becomes equivalent to the usual prescription for introducing a "momentum" dependence that is associated with the group index. \&

We take $P_{\mu}$ to be diagonal

$$
P_{\mu}=\left(\begin{array}{cccc}
p_{\mu}^{1} & 0 & & \\
0 & p_{\mu}^{2} & & \\
& & \ldots & \\
& & & p_{\mu}^{N}
\end{array}\right)
$$

where $P_{\mu}^{i}$ belong to the set of eigenvalues of momentum in the space-time of interest, and assumed to be uniformly distributed. Then we recover the YM action in the quenched momentum prescription, with a kinetic term and cubic and quartic interaction vertices.

$$
S=\left(\frac{2 \pi}{\Lambda}\right)^{d} \frac{1}{2 g^{2}}\left\{\left[P_{\mu}, a_{\nu}\right]^{2}+2\left[P_{\mu}, a_{\nu}\right]\left[a_{\mu}, a_{\nu}\right]+\left[a_{\mu}, a_{\nu}\right]^{2}\right\}
$$

We also have to include gauge fixing terms as in [15, 16] but for our purposes the above action will suffice. Finally we can use

$$
\frac{1}{N}\left(\frac{2 \pi}{\Lambda}\right)^{d} \sum_{i=1}^{N} f\left(p_{\mu}^{i}\right) \rightarrow \int_{-\frac{\Lambda}{2}}^{+\frac{\Lambda}{2}} \frac{d^{d} p}{(2 \pi)^{d}} f\left(p_{\mu}^{i}\right)
$$

We also have the following relations:

$$
P=V_{3}+V_{4}+L-1
$$

\footnotetext{
${ }^{2}$ As mentioned above, the $A_{\mu}$ have the interpretation of position of the D-branes. In the context of the quenched reduced models [12, 13, 14, 15, 16, however, this would correspond to momentum.
} 


$$
2 P=3 V_{3}+4 V_{4}
$$

where $P, V_{3}, V_{4}, L$ are the number of propagators, cubic and quartic vertices and loops respectively. Thus keeping in mind the following planar diagram (Figure 1) for concreteness, and using the fact that there are factors of $g\left(\frac{2 \pi}{\Lambda}\right)^{d}$ associated with a cubic vertex, $g^{2}\left(\frac{2 \pi}{\Lambda}\right)^{d}$ with a quartic vertex and $\frac{1}{\left(p_{i}-p_{j}\right)^{2}\left(\frac{2 \pi}{\Lambda}\right)^{d}}$ with a propagator, we get

$$
\left(g^{2}\right)^{\frac{V_{3}}{2}+V_{4}}\left[\left(\frac{2 \pi}{\Lambda}\right)^{d}\right]^{V_{3}+V_{4}-P} \underbrace{\sum_{i, j \ldots}}_{L+1} f\left(p^{i}, p^{j}, \ldots\right)
$$

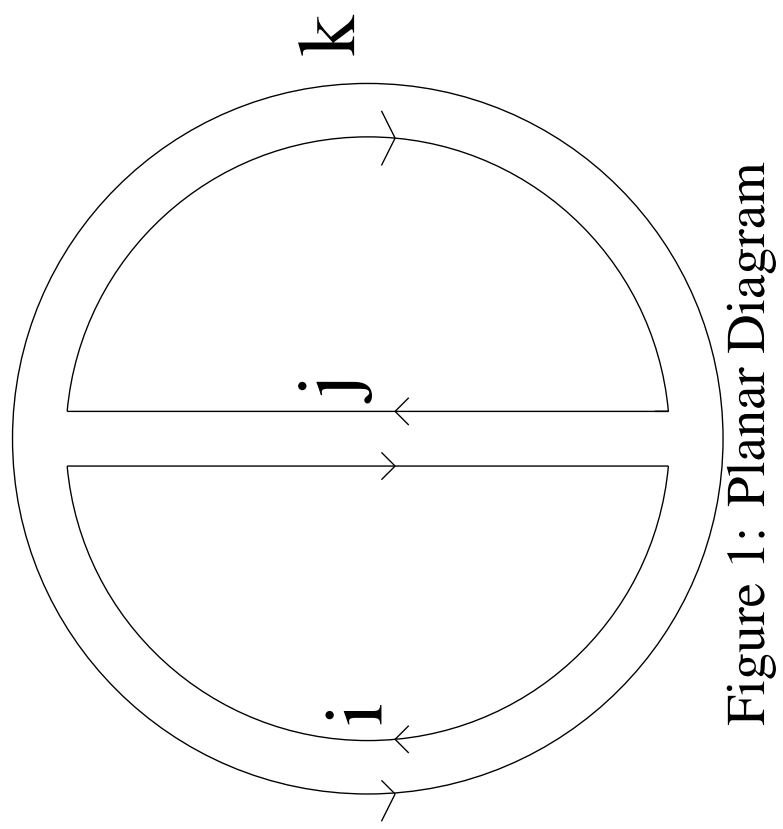

Now we replace $\Sigma_{i}$ by $\left(\frac{2 \pi}{\Lambda}\right)^{d} N \int_{-\frac{\Lambda}{2}}^{+\frac{\Lambda}{2}} \frac{d^{d} p}{(2 \pi)^{d}}$. Since only differences of the form $p_{i}-p_{j}$ are involved, there is one overall factor of $N$, not accompanied by any momentum integration. This gives

$$
\begin{gathered}
N^{L+1}\left[\left(\frac{2 \pi}{\Lambda}\right)^{d}\right]^{L}\left(g^{2}\right)^{L-1}\left[\left(\frac{2 \pi}{\Lambda}\right)^{d}\right]^{(1-L)} \underbrace{\int_{-\frac{\Lambda}{2}}^{+\frac{\Lambda}{2}} \frac{d^{d} p}{(2 \pi)^{d}} \ldots}_{L} f\left(p_{i} \ldots\right) \\
=N^{2}\left(g^{2} N\right)^{L-1}\left(\frac{2 \pi}{\Lambda}\right)^{d}[\text { Usual Feynman diagram }+O(1 / N)]
\end{gathered}
$$


By usual Feynman diagram, we mean that of the unreduced model. The $\frac{1}{N}$ corrections are due to the fact that in some of the terms in the sum (2.9), the propagator may have zero momentum, being of the form " $p_{i}-p_{j}$ " with $i=j$. In our case, since we are not concerned with separating out a piece relevant to the unreduced model, the full expression in square brackets is the answer. The overall volume factor of $\left(\frac{2 \pi}{\Lambda}\right)^{d}$ is expected and one divides it out to get the free energy per unit volume.

We can now consider a non planar diagram with one handle as in Figure 2 .

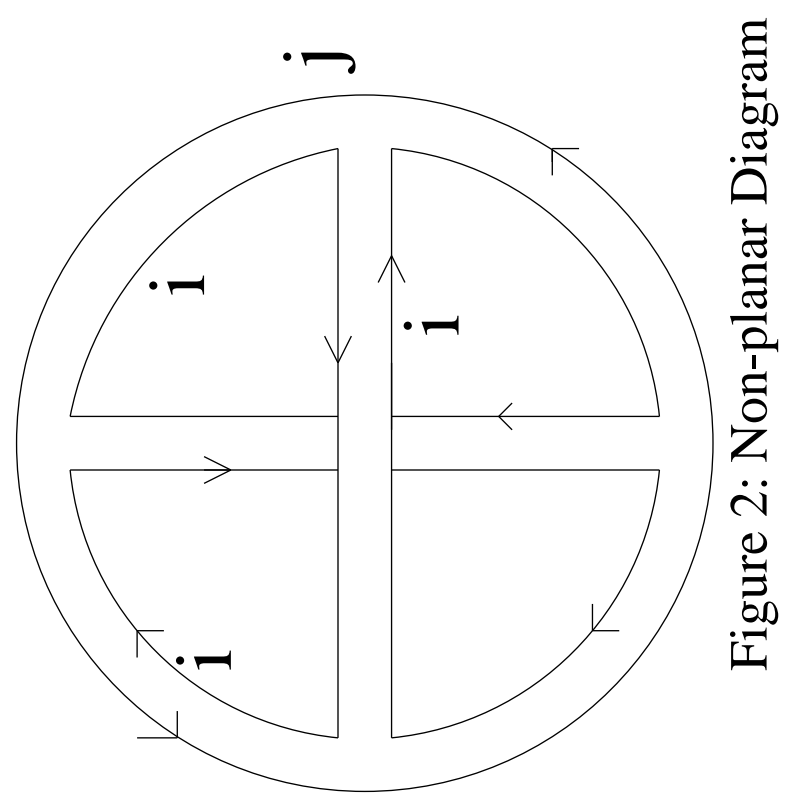

Note that while there are three loops in the usual sense there are only two index loops. Thus we get a $N^{L-1}$ rather than $N^{L+1}$. In general we get $N^{L+1-2 H}$ where $H$ is the number of handles. Since momentum integration is associated with the group index, there are only two of them $: \sum_{i} \sum_{j}$ and again since only $p_{i}-p_{j}$ is involved, we effectively have one integration. Thus $\sum_{i} \sum_{j} f\left(p_{i}, p_{j}\right)=N^{2}\left(\frac{2 \pi}{\Lambda}\right)^{d} \int_{-\frac{\Lambda}{2}}^{+\frac{\Lambda}{2}} \frac{d^{d} p}{(2 \pi)^{d}} f(p)$, for this particular example. In general it is $N^{L+1-2 H}\left[\left(\frac{2 \pi}{\Lambda}\right)^{d}\right]^{L-2 H}[\underbrace{\left[\int_{-\frac{\Lambda}{2}}^{+\frac{\Lambda}{2}} \frac{d^{d} p}{(2 \pi)^{d}} \ldots\right]}_{L-2 H \text { of them }} f(p)$. 
Thus for a non planar diagram (2.11) gets replaced by

$$
N^{2}\left(g^{2} N\right)^{L-1}\left[N\left(\frac{2 \pi}{\Lambda}\right)^{d}\right]^{-2 H}[\text { Momentum integral }]
$$

The expression in square brackets has fewer momenta integrated over and is therefore more convergent than that of the leading term (2.11). If we assume that these integrals are convergent for reasons of supersymetry and that the perturbation makes sense, then we see clearly that the genus expansion parameter is not $\frac{1}{N}$ but $\frac{1}{N\left(\frac{2 \pi}{\Lambda}\right)^{d}}$. Thus we can take $N$ to $\infty$, along with $\Lambda^{d}$ (keeping $g^{2} N$ fixed as usual) and get a non zero contribution from the non planar diagrams. We can thus identify the string coupling constant $g_{s}$

$$
g_{s}=\frac{1}{N\left(\frac{2 \pi}{\Lambda}\right)^{d} m^{d}}
$$

where " $m$ " is some finite mass scale characterizing the theory. This is essentially the double scaling limit advocated in [2], with $d=10$.

However, there is an ambiguity here. We could also imagine starting with the same model in a lower spacetime dimension. This corresponds to giving an expectation value to only some of the $A_{\mu}$. This should then correspond to a reordering of the perturbation series where the propagator is expanded out, viz:

$$
\frac{1}{\sum_{\mu=1}^{10} p^{\mu} p_{\mu}}=\frac{1}{\sum_{i=1}^{d} p^{i} p_{i}}\left[1+\frac{\sum_{j=d+1}^{10} p^{j} p_{j}}{\sum_{i=1}^{d} p^{i} p_{i}}+\ldots\right]
$$

The numerators in the above series correspond to the effects of quartic interaction vertices where two of the $A_{\mu}$ take vevs $\left\langle A_{i}\right\rangle=p_{i}$. In particular one could start with $d=2$ and in that case we would interpret it as the string world sheet. The full space time interpretation would emerge on calculating string diagrams with sources ("vertex operators") that have the effect of the non leading terms in (2.14). Presumably, since we are only reordering perturbation theory, one should still have the original double scaling with $\frac{N}{\Lambda^{10}}$. But in each individual graph this would not make sense - one would need $N\left(\frac{2 \pi}{\Lambda}\right)^{d}$. One possible explanation is that the double scaling should be chosen according to the vacuum that one is expanding about and different limits correspond to exploring different vacua. We have to also keep in mind that the space-time interpretation in string theory requires the " $p$ " to be position coordinates. In the Yang Mills description they are momenta. 
To conclude this section, if we accept the conjecture that the $d=10$ SSYM defines string theory and that perturbation about flat 10-d space time makes sense, then the double scaling prescription (2.13) of IKKT seems to be the right one. 


\section{World Sheet Coordinates for the Funda- mental String and the D-string}

If we accept that the large $N$ reduced SSYM action of [2] describes the IIB theory we can write the action as

$$
S=\frac{T r}{\alpha^{\prime 2} g_{s}}\left\{\left[A^{\mu}, A^{\nu}\right]^{2}+\bar{\Psi} \Gamma^{\mu} A_{\mu} \Psi\right\}
$$

Noting that the 10-dimensional gravitational constant $\kappa \approx \alpha^{\prime 2} g_{s}$ we can rewrite (3.1) as

$$
S=\frac{1}{\kappa} \operatorname{Tr}\left[A^{\mu}, A^{\nu}\right]^{2}+\text { Fermionic }
$$

One advantage of introducing $\kappa$ in place of $\alpha^{\prime 2} g_{s}$ is that it looks more duality invariant - it does not give a special role to $\alpha^{\prime}$. This is particularly important for type IIB where there is a complete duality between F-strings and D-strings. While this is a non perturbative result, we should expect to see this symmetry in the matrix model formulation which purports to being a non perturbative definition of string theory. While keeping this requirement in mind, following the suggestion of IKKT that the Wilson loop should correspond to the $\mathrm{F}$ string, let us introduce world sheet coordinates $\tau_{F}$ and $\sigma_{F}$ for the $\mathrm{F}$-string by giving expectation values to $A^{0}$ and $A^{1}$. This forces us into the light cone gauge.

We use the fact that a general $N \times N$ matrix can be expanded in a basis.

$$
A=\sum_{m, n} A_{m n} e^{i m p} e^{i n q}
$$

where

$$
[q, p]=\frac{2 \pi i}{N}
$$

There is the usual caveat that $N$ has to be infinity for this to work. To make things concrete, consider a particle in a one dimensional box of length $L$, with periodic boundary conditions. Also assume an ultraviolet cutoff i.e. a lattice spacing " $a$ ". The number of sites is $L / a \equiv N$. We introduce canonical

\footnotetext{
22].

${ }^{3}$ We have not discussed the fermion sector in this paper. This will be reported elsewhere
} 
momentum and position operators $P$ and $Q$. The momentum eigenfunctions are of the form

$$
\phi_{n}=\frac{1}{\sqrt{L}} e^{i p_{n} x}
$$

where $p_{n}=\frac{2 \pi n}{L}=\frac{2 \pi n}{N} a$ is the $n^{\text {th }}$ eigenvalue of the momentum operator $P$. Let us choose $a=\sqrt{\frac{2 \pi}{N}}$ and $L=\sqrt{N \pi}$. Then

$$
p_{n}=\frac{2 \pi n}{\sqrt{N 2 \pi}} 0 \leq n \leq N-1
$$

The operator $p=P \sqrt{\frac{2 \pi}{N}}$, has eigenvalues $\frac{2 \pi n}{N}$. The matrix $e^{i P \sqrt{\frac{2 \pi}{N}}}$ then is

$$
e^{i p}=e^{i P \sqrt{\frac{2 \pi}{N}}}=\left(\begin{array}{ccccc}
1 & 0 & & & \\
0 & \omega & & & \\
& & & 0 & \\
& & 0 & \cdots & \\
& & & \omega^{N-1}
\end{array}\right)
$$

where $\omega$ are the $N^{t h}$ roots of unity. $Q$ has possible values $0, a, 2 a, \ldots, n a, . .(N-$ 1) $a$ which is the same as

$$
\left(0, \frac{1}{\sqrt{N}}, \ldots ., \frac{N-1}{\sqrt{N}}\right) \sqrt{2 \pi}
$$

the same as $P$. In this normalization $[Q, P]=i$. If I choose $q=\sqrt{\frac{2 \pi}{N}} Q$ so that

$$
[q, p]=\frac{2 \pi i}{N}
$$

it has eigenvalues $\frac{n}{N} 2 \pi$, the same as $p$.

$$
e^{i q}=\left(\begin{array}{ccccc}
0 & 1 & & & \\
0 & 0 & 1 & & \\
& & 0 & 1 & \\
& & & . \cdot & 1 \\
1 & & & & 0
\end{array}\right)
$$

Note again that the matrix $q$ satisfying (3.9) and (3.10) only exist in the limit $N \rightarrow \infty$ which is also the limit $L \rightarrow \infty$ or $a \rightarrow 0$. 
We introduce $\tau_{F}$ and $\sigma_{F}$ by the following ansatz

$$
\begin{aligned}
& {\left[A^{0}\right]_{i a}^{j b}=\frac{1}{\sqrt{2}} \kappa^{1 / 4} g_{s}^{1 / 4}\left[-i \frac{\partial}{\partial \tau_{F}}\right]_{i}^{j} \delta_{a}^{b}+\left[a^{0}\right]_{i a}^{j b}} \\
& {\left[A^{1}\right]_{i a}^{j b}=\frac{1}{\sqrt{2}} \kappa^{1 / 4} g_{s}^{1 / 4}\left[-i \frac{\partial}{\partial \sigma_{F}}\right]_{a}^{b} \delta_{i}^{j}+\left[a^{1}\right]_{i a}^{j b}}
\end{aligned}
$$

$i, j=1 \ldots \ldots N_{1} a, b=1, N_{2}$. We can set

$$
\begin{aligned}
& 2 \pi \tau_{F}=q_{1} ;-i \frac{\partial}{\partial \tau_{F}}=N_{1} p_{1} \\
& 2 \pi \sigma_{F}=q_{2} ;-i \frac{\partial}{\partial \sigma_{F}}=N_{2} p_{2}
\end{aligned}
$$

One can, if one wants, let $2 \pi \tau_{F}=N_{1} q_{1}$ and let $-i \partial_{\tau_{F}}=p_{1}$ and remove the factor $N_{1}$ in (3.14). This may be more convenient for some purposes. However, in this paper we have treated $\sigma$ and $\tau$ symmetrically.

Here $a^{0}$ and $a^{1}$ are fluctuations about the assumed background. Thus

$$
\begin{aligned}
& <A^{0}>=\frac{1}{\sqrt{2}} \kappa^{1 / 4} g_{s}^{1 / 4}\left[p_{1}\right]_{i}^{j} \delta_{a}^{b} N_{1} \\
& <A^{1}>=\frac{1}{\sqrt{2}} \kappa^{1 / 4} g_{s}^{1 / 4}\left[p_{2}\right]_{a}^{b} \delta_{i}^{j} N_{2}
\end{aligned}
$$

The action (3.2) becomes $\left[N_{1} N_{2}=N\right]$

$$
S=\frac{\sqrt{g_{s}}}{2 \sqrt{\kappa}}\left[\left(F_{01}\right)^{2}+\left[D_{\tau F} A^{I}\right]^{2}+\left[D_{\sigma F} A^{I}\right]^{2}\right]+\frac{1}{\kappa} \sum_{I<J}\left[A^{I}, A^{J}\right]^{2}
$$

Here $D_{\tau F}$ and $D_{\sigma F}$ are covariant derivatives in the $\tau_{F}$ and $\sigma_{F}$ directions. If we ignore the interaction terms (and $F_{01}$ ) and assume that $A^{I}$ is only a function of $\tau_{F}$ and $\sigma_{F}$, then they are like the transverse string coordinates. This is the light cone gauge string action. We will also work out, in the next section, the two point function for a Wilson loop and show that the Wilson loop is nothing but a vertex operator.

If we look at (3.16) it also looks like the action that describes the fluctuations of a D-string, except for the factor $\sqrt{\frac{g_{s}}{\kappa}}\left(=\frac{1}{\alpha^{\prime}}\right)$ which reminds us that this is supposed to be a fundamental string action. Before we worry about 
this source of confusion, let us consider the classical D-string solution of [2] In our language it corresponds to turning on an electric field by giving an expectation value to $a^{0}$ and $a^{1}$ in (3.11) and (3.12) $)^{\text {. Thus }}$. The let

$$
\begin{gathered}
<a_{0}>=\frac{1}{\sqrt{2}} \kappa^{1 / 4} g_{s}^{-1 / 2} \frac{\sigma_{F a}^{b} \delta_{i}^{j}}{\left(N_{1} N_{2}\right)^{1 / 4}}=\frac{1}{\sqrt{2}} \kappa^{1 / 4} g_{s}^{-1 / 2} \frac{q_{2 a}^{b} \delta_{i}^{j}}{2 \pi\left(N_{1} N_{2}\right)^{1 / 4}} \\
<a_{1}>=-\frac{1}{\sqrt{2}} \kappa^{1 / 4} g_{s}^{-1 / 2} \frac{\tau_{F i}^{j} \delta_{a}^{b}}{\left(N_{1} N_{2}\right)^{1 / 4}}=-\frac{1}{\sqrt{2}} \kappa^{1 / 4} g_{s}^{-1 / 2} \frac{q_{1 i}^{j} \delta_{a}^{b}}{2 \pi\left(N_{1} N_{2}\right)^{1 / 4}}
\end{gathered}
$$

Thus $<A^{0}>_{\text {total }}$ is now the sum of (3.14) and (3.17) and similarly for $<A^{1}>_{\text {total }} .<A^{0}>_{\text {total }}$ and $<A^{1}>_{\text {total }}$ do not commute now and

$$
\begin{gathered}
{\left[<A^{0}>_{\text {total }},<A^{1}>_{\text {total }}\right]=F_{01}=i \frac{\sqrt{\kappa} g_{s}^{-1 / 4} \delta_{i}^{j} \delta_{a}^{b}}{\left(N_{1} N_{2}\right)^{1 / 4}}} \\
S_{\text {classical }}=\frac{T r}{\kappa}\left(F_{01}\right)^{2}=\frac{\sqrt{N_{1} N_{2}}}{\sqrt{g_{s}}}=\frac{T L}{\sqrt{\kappa g_{s}}}
\end{gathered}
$$

We have set $\sqrt{N_{1}}=T$ and $\sqrt{N_{2}}=L$, both in units of $\kappa^{1 / 4}$. (Note that $Q_{1,2}$, which is the one that has canonical commutation relations, has a range of $\sqrt{2 \pi N_{1,2}}$ whereas the world sheet coordinate $q_{1,2}$ has a range of $2 \pi$.) This gives a tension of $\mathrm{O}\left(\frac{1}{\sqrt{g_{s}}}\right)$ in Planck units, as required for D-strings. In this picture fluctuations (that depend on $\tau_{F}, \sigma_{F}$ ) about this classical solution are fundamental strings.

Now we notice something interesting. $p$ and $q$ have the same spectrum. We could have let

$$
<A^{0,1}>=\frac{1}{\sqrt{2}}\left(\frac{\kappa}{g_{s}}\right)^{1 / 4} q_{1,2} N_{1,2}
$$

instead of $p_{1,2}$ as in (3.14), 3.15). The entire analysis would have gone through in exactly the same way. We can introduce worldsheet coordinates $\tau_{D}$ and $\sigma_{D}$ and set

$$
\begin{aligned}
& N_{1} q_{1}=-i \frac{\partial}{\partial \tau_{D}} ; \quad p_{1}=2 \pi \tau_{D} \\
& N_{2} q_{2}=-i \frac{\partial}{\partial \sigma_{D}} ; \quad p_{2}=2 \pi \sigma_{D}
\end{aligned}
$$

\footnotetext{
${ }^{4}$ Similar observations have been made in $[$ ⿹ precise contact
} 
We get an action identical to (3.16) with the subscript F replaced by D and having an inverse string tension $\beta^{\prime}=\alpha^{\prime} g_{s}$ :

$$
S=\frac{1}{2 \sqrt{\kappa g_{s}}}\left[\left(F_{01}\right)^{2}+\left[D_{\tau D} A^{I}\right]^{2}+\left[D_{\sigma D} A^{I}\right]^{2}\right]+\frac{1}{\kappa} \sum_{I<J}\left[A^{I}, A^{J}\right]^{2}
$$

As before, $D_{\tau D}$ and $D_{\sigma D}$ are covariant derivatives in the $\tau_{D}$ and $\sigma_{D}$ directions. We interpret this as the D-string action. We have to impose that $A^{I}$ are functions of $\tau_{D}$ and $\sigma_{D}$ in contrast to the F-string configurations which were functions of $\tau_{F}$ and $\sigma_{F}$. Thus the interchange of $p \leftrightarrow q$ and $g_{s} \leftrightarrow \frac{1}{g_{s}}$ is the $\mathrm{SL}(2, \mathrm{Z})$ duality that interchanges the $\mathrm{F}$ and D-strings. Note that

$$
\left(\begin{array}{l}
p^{\prime} \\
q^{\prime}
\end{array}\right)=\left(\begin{array}{ll}
a & b \\
c & d
\end{array}\right)\left(\begin{array}{l}
p \\
q
\end{array}\right)
$$

preserves the commutation relations, provided $a d-b c=1$. Since $p$ and $q$ have identical discrete spectra, $a, b, c, d$ have to be integers.

In Equation (3.16) small fluctuations about a classical solution are described by variables $a^{0,1}, A^{I}$. In general they can be functions of $q$ and $p$, i.e. functions of $\sigma_{F}, \tau_{F}$ as well as $\frac{\partial}{\partial \tau_{F}}, \frac{\partial}{\partial \sigma_{F}}$, which is what we have been calling $\tau_{D}$ and $\sigma_{D}$. Fluctuations that are purely functions of $\left(\tau_{F}, \sigma_{F}\right)$ or $\left(\tau_{D}, \sigma_{D}\right)$ are F-strings and D-strings respectively. If we were to substitute into the quartic interaction term, the most general configuration, we would get terms that, viewed from the F-string (or D-string) world sheet perspective, involve higher derivatives in $\tau, \sigma$. These look somewhat like massive mode backgrounds and should play a part in determining the superstring interactions.

The viewpoint presented here is not specific to IIB and applies to IIA also. However IIB has an exact SL(2,Z) symmetry and the above observations are more readily applicable. Thus to summarize, if the above interpretation is correct, then it is very simple to consider F and D-string configurations on the same footing at the same time and duality is manifest. The classical solutions break the symmetry through particular choices of the expectation values of the matrices, which are chosen to reproduce either D- or F- string backgrounds. These solutions are distinguished only by the relative amounts of $p$ and $q$ in the expectation value of $A$.

Given a classical D-string background (3.16), one can quantize collective fluctuations about this background. The Wilson loop variables $\delta a_{1} L$ are like one dimensional rotors with quantized energies. As pointed out in $18 \pi e^{i n a_{1} L}$ 
are eigenstates of the electric field operator. The energy is of $\mathrm{O}\left(g_{s}\right)$ (in Fstring units). These are thus the $(n, 1)$ strings. If we start with a multiply wound classical background one should get the (n,m) strings. It should not be too hard to write everything in a manifestly $\mathrm{SL}(2, \mathrm{Z})$ covariant manner to reproduce the spectrum of BPS states as done in 19. 


\section{Wilson Loop and Vertex Operators}

In $(\overline{3.16})$, the action for the transverse $A^{I}$ is

$$
\frac{\operatorname{Tr}}{\kappa}\left\{\left[<A^{0}>, A^{I}\right]^{2}+\left[<A^{1}>, A^{I}\right]^{2}+\sum_{I<J}\left[A^{I}, A^{J}\right]^{2}\right\}
$$

If we use (3.14)

$$
\begin{aligned}
& <A^{0}>=\frac{1}{\sqrt{2}} \kappa^{1 / 4} g_{s}^{1 / 4}\left[p_{1}\right]_{i}^{j} \delta_{a}^{b} N_{1} \\
& <A^{1}>=\frac{1}{\sqrt{2}} \kappa^{1 / 4} g_{s}^{1 / 4}\left[p_{2}\right]_{a}^{b} \delta_{i}^{j} N_{2}
\end{aligned}
$$

It is easiest to expand $A^{I}$ as

$$
A^{I}=\sum_{m, n, r, s} A_{m n r s} e^{i m p_{1}} e^{i n q_{1}} e^{i r p_{2}} e^{i s q_{2}}
$$

Using $\left[q_{1}, p_{1}\right]=\frac{2 \pi i}{N_{1}}$

$$
\left[<A^{0}>, A^{I}\right]=\frac{\kappa^{1 / 4} g_{s}^{1 / 4}}{\sqrt{2}} \sum_{m, n, r, s} A_{m n r s} 2 \pi n e^{i m p_{1}} e^{i n q_{1}} e^{i r p_{2}} e^{i s q_{2}}
$$

This is to be plugged into the action. To evaluate the trace we introduce the basis vectors $\mid \tilde{q}>$, with $\langle\tilde{q}| \tilde{q}>=1$ and $\left|\tilde{p}>=\sum_{\tilde{q}} e^{i \tilde{p} \tilde{q}}\right| \tilde{q}>$,

$$
<\tilde{p} \mid \tilde{p}>=\sum_{q} 1=N_{1}
$$

Thus

$$
\begin{gathered}
\operatorname{Tr}\left[<A^{0}>, A^{I}\right]^{2}= \\
-2 \pi^{2} \sqrt{g_{s} \kappa} N_{1} N_{2} \sum_{m, n, r, s} n^{2} A_{m n r s} A_{-m-n-r-s} e^{2 \pi i \frac{m n}{N_{1}}} e^{2 \pi i \frac{r s}{N_{2}}}
\end{gathered}
$$

Similarly

$$
\begin{gathered}
\operatorname{Tr}\left[<A^{1}>, A^{I}\right]^{2}= \\
-2 \pi^{2} \sqrt{g_{s} \kappa} N_{1} N_{2} \sum_{m, n, r, s} r^{2} A_{m n r s} A_{-m-n-r-s} e^{2 \pi i \frac{m n}{N_{1}}} e^{2 \pi i \frac{r s}{N_{2}}}
\end{gathered}
$$


The quadratic part of the action becomes

$$
-2 \pi^{2} \sqrt{\frac{g_{s}}{\kappa}} N_{1} N_{2} \sum_{m, n, r, s}\left(n^{2}+r^{2}\right) A_{m n r s} A_{-m-n-r-s} e^{2 \pi i \frac{m n}{N_{1}}} e^{2 \pi i \frac{r s}{N_{2}}}
$$

Thus

$$
<<A_{m n r s} A_{a b c d}>>=\sqrt{\frac{\kappa}{g_{s}}} \frac{\delta_{m+a} \delta_{n+b} \delta_{r+c} \delta_{s+d} e^{-2 \pi i \frac{m n}{N_{1}}} e^{-2 \pi i \frac{r s}{N_{2}}}}{2 \pi^{2} N_{1} N_{2}\left(r^{2}+n^{2}\right)}
$$

is the two point function.

\subsection{Two point function for the Wilson Loop}

The Wilson loop in this model can be defined as p

$$
\operatorname{Tr} e^{K A}=\sum_{i a}\left[e^{K A}\right]_{i a}^{i a}
$$

where $K_{i a}^{j b}$ is a general $N_{1} N_{2} \times N_{1} N_{2}$ matrix. Let us calculate

$$
<\operatorname{Tr} e^{K A} \operatorname{Tr} e^{\tilde{K} A}>
$$

Expanding each exponential and using the expansion (4.4) for both $K$ and $A$, and also using

$$
<q\left|e^{i\left(m+m^{\prime}\right) p} e^{i\left(n+n^{\prime}\right) q}\right| q>=e^{i\left(n+n^{\prime}\right) q} \delta_{m+m^{\prime}}
$$

we get to lowest order (suppressing the indices on $p, q, N$, so that each of the $m, n$. are two dimensional vectors)

$$
\sum_{q, q^{\prime}} \sum_{m, n, m^{\prime}, n^{\prime}} \sum_{r, s, r^{\prime}, s^{\prime}} K_{m^{\prime} n^{\prime}} \tilde{K}_{r^{\prime} s^{\prime}}<<A_{m n} A_{r s}>>e^{i\left(n+n^{\prime}\right) q} \delta_{m+m^{\prime}} e^{i\left(s+s^{\prime}\right) q} \delta_{r+r^{\prime}} e^{2 \pi i \frac{r^{\prime} m}{N}} e^{2 \pi i \frac{s^{\prime} r}{N}}
$$

Let us also set

$$
K_{m^{\prime} n^{\prime}}=k \delta_{m^{\prime}, 0} \delta_{n^{\prime}, 0} \quad ; \quad \tilde{K}_{r^{\prime} s^{\prime}}=\tilde{k} \delta_{r^{\prime}, 0} \delta_{s^{\prime}, 0}
$$

\footnotetext{
${ }^{5}$ The name "Wilson Loop" is a misnomer, since for generic $K$, it is not invariant under $S U(N)$ transformations. However for want of a better name we will refer to it as a Wilson loop.
} 
as a special choice. We find (4.14) becomes

$$
k . \tilde{k} \sum_{q, q^{\prime}} \sum_{n} \frac{e^{i n\left(q-q^{\prime}\right)}}{2 \pi^{2} N n^{2}}
$$

Restoring the two dimensional nature we see that we have in the continuum limit:

$$
k . \tilde{k} \int d^{2} q \int d^{2} q^{\prime} \frac{\ln \left(q-q^{\prime}\right)}{N}
$$

At the next order we get

$$
(k . \tilde{k})^{2} \int d^{2} q \int d^{2} q^{\prime} \frac{\ln ^{2}\left(q-q^{\prime}\right)}{2 N}
$$

Thus it is easy to see that it exponentiates to give:

$$
<\operatorname{Tr} e^{K A} \operatorname{Tr} e^{\tilde{K} A}>=\int d^{2} q \int d^{2} q^{\prime} e^{k \cdot \tilde{k} \ln \left(q-q^{\prime}\right)}
$$

Thus we see that $\operatorname{Tr} e^{K A}$ behaves like $e^{i k X}$ in ordinary string theory. IIt should be possible to reproduce the rest of the vertex operators after imposing supersymmetry.

\subsection{One point function for Wilson loop in a D-string background}

Let us now turn to the one point function of a general Wilson loop operator in the presence of a D-string. This was evaluated also in [2]. The D-string background can be written as

$$
\begin{gathered}
A^{0}=a p_{1}+b q_{2} \\
A^{1}=c p_{2}+d q_{1} \\
A^{I}=x^{I}
\end{gathered}
$$

for some $a, b, c, d$ and $x^{I}$ is a constant matrix specifying the location of the D-string which is in the $x^{1}$ direction.

\footnotetext{
${ }^{6}$ Note that the integral over q,q' will reproduce the spacetime propagator for that mode of the string.
} 
We consider a Wilson loop representing a general closed string configuration:] $\operatorname{Tr}^{K A}$. The closed string world sheet coordinates being $q_{1}=\tau_{F}$ and $q_{2}=\sigma_{F}$, we can assume that $K^{\mu}$ is only a function of $q_{2}, p_{2}$. Thus

$$
\operatorname{Tr} e^{K A}=\int d p_{1} d p_{2} d q_{1} d q_{2} e^{K^{0}\left(q_{2}, p_{2}\right)\left(a p_{1}+b q_{2}\right)+K^{1}\left(q_{2}, p_{2}\right)\left(c p_{2}+d q_{1}\right)+K^{I}\left(q_{2}, p_{2}\right) x^{I}}
$$

The $p_{1}$ and $q_{1}$ integrals clearly give $\delta\left(K^{0}\left(q_{2}, p_{2}\right)\right) \delta\left(K^{1}\left(q_{2}, p_{2}\right)\right)$. $K^{I}\left(q_{2}, p_{2}\right)$ contains, in addition to the zero mode $K_{0}^{I}$, which just gives a factor $e^{i K_{0}^{I} x^{I}}$, modes corresponding to the left and right movers. The integral over $q_{2}$ imposes the $L_{0}=\bar{L}_{0}$ constraint. Without knowing the precise form of $K$ it is difficult to say more. For instance if there are necessarily factors of $p_{2}$ accompanying the non-zero modes, it will clearly give zero acting on $x^{I}$ which is proportional to the identity.

The delta functions in the 0 and 1 directions imply that $\partial_{\tau_{F}} X^{0}=\partial_{\tau_{F}} X^{1}=$ 0 for the closed string. By world sheet duality (s-channel - t-channel) this is equivalent to $\partial_{\sigma_{F}} X^{0}=\partial_{\sigma_{F}} X^{1}=0$ for the open string attached to the Dbrane. Similarly the factor $e^{i k_{0} x^{I}}$ signifies the Dirichlet boundary conditions in the other directions. This calculation can thus be viewed as a matrix model derivation of the boundary conditions that define a D-string in the conventional approach [20].

\footnotetext{
${ }^{7}$ The continuum representation should be equivalent to the 'loop variables' introduced in 21 .

${ }^{8}$ This is analogous to the fact that $e^{i k X(\tau)}$ is the vertex operator for a scalar particle where the momentum $k$ does not depend on $\tau$.
} 


\section{Conclusions}

In this paper we have attempted to explore some of the consequences of the proposal of [2] identifying the reduced large N (10 - dim) SSYM as a non-perturbative definition of type IIB superstring. We showed that if we consider the Feynman diagrams of the quenched reduced model, a sensible genus expansion can be defined provided a double scaling limit is taken. The

small parameter is not $\frac{1}{N}$ but $\frac{1}{N\left(\frac{2 \pi}{\Lambda}\right)^{d}}$, where $\Lambda$ is proportional to the volume of spacetime. A power of $m^{d}$ must be included for dimensional reasons, $m$ being some mass parameter of the full theory. The choice $d=10$, reproduces the scaling proposed by [2]. This parameter can thus be identified with $g_{s}$ and the Wilson loop becomes the fundamental string. We then introduced world sheet coordinates $\sigma_{F}, \tau_{F}$ for the fundamental string and argued that the Dstring coordinates are essentially the conjugates $\partial_{\sigma_{F}}, \partial_{\tau_{F}}$ upto some overall normalization. The $\mathrm{SL}(2, \mathrm{Z})$ duality then becomes the usual symplectic group of transformations acting on $(\mathrm{p}, \mathrm{q})$. The classical D-string coresponds to turning on a background electric field. By exchanging $p \leftrightarrow q$ and $g_{s} \leftrightarrow \frac{1}{g_{s}}$, one can also get a classical string solution. The collective coordinate fluctuations can be quantized to get the $(m, n)$ strings. We have not done this in a manifestly $\mathrm{SL}(2, \mathrm{Z})$ invariant manner, but it should be possible to do so in this formalism. Finally we have identified the Wilson loop equivalent of the vertex operator $e^{i k X}$ and shown that the two point function has the right form. We have also calculated the one point function of a general closed string vertex operator in the presence of a D-string. This is tantamount to a derivation, within the matrix model of the Dirichlet and Neumann boundary conditions that define D-branes in the usual approach.

There are many questions that need to be answered. One principal issue is that of interactions. This is to be derived from the quartic term $\left[A^{I}, A^{J}\right]^{2}$. Since $\left[p_{i}, q_{i}\right] \approx \frac{1}{N_{i}}$ and $N_{1} N_{2}=N=\frac{1}{g_{s}}$, we can naturally expect the coupling constant to show up here. The details need to be worked out, to see whether it agrees with the IIB superstring. This has been investigated in the context of the IIA string in [5]. Finally, most of the formalism of this paper can be applied to the IIA matrix model. This also needs to be worked out.

\section{Acknowledgements}

I would like to thank T. Jayaraman, G. Sengupta, N. Hari Dass and R. Kaul for useful discussions. I would also like to thank T. Jayaraman and N. Hari 
Dass for a careful reading of the manuscript and for useful suggestions. 


\section{References}

[1] T. Banks, W. Fischler, S. Shenker and L. Susskind, hepth/9610043.

[2] N.Ishibashi, H. Kawai, Y. Kitazawa and A. Tsuchiya, hepth/9612115.

[3] L. Motl, hepth/9701025.

[4] T. Banks and N. Seiberg, hepth/9702187.

[5] R. Dijkgraaf, E. Verlinde and H. Verlinde, hepth/9703030.

[6] Y. Imamura, hepth/9703077.

[7] I. Chepelev, Y. Makeenko and K. Zarembo, hepth/970115.

[8] A. Fayyazuddin, Y. Makeenko, P. Olesen, D. J. Smith, and K. Zarembo, hepth/9703038.

[9] A. Fayyazuddin and D. J. Smith, hepth/ 9701168.

[10] M. Li, hepth/ 9612222.

[11] T. Yoneya, hepth/9703078.

[12] T. Eguchi and H. Kawai, Phys. Rev. Lett. 48 (1982) 1063.

[13] G. Bhanot, U. Heller, and H. Neuberger, Phys. Lett. 113B (1982) 47.

[14] G. Parisi, Phys. Lett 112B (1982)463.

[15] S. Das and S. Wadia, Phys. Lett. 117B (1982) 367.

[16] D. Gross and Y. Kitazawa, Nucl. Phys. B206 (1982) 440.

[17] I. Bars, Phys. Lett. 245B (1990) 35.

[18] E. Witten, hepth/9510135.

[19] J. Schwarz, hepth/9508143.

[20] J. Polchinski, hepth/9510017.

[21] B. Sathiapalan, Nucl. Phys. B326 (1989) 376.

[22] T. Jayaraman and B. Sathiapalan, in preparation. 